\title{
La toma de decisiones de rescatistas y la efectividad en la primera ayuda psicológica
}

\author{
The decision making of the rescuers and effectiveness
}

in the first psychological aid

\author{
Aldana Sol Grinhauz 1 , Sergio H. Azzara ${ }^{2}$, Alejandro M. Otamendi ${ }^{3}$ \\ y Susana C. Azzollini ${ }^{4}$
}

\begin{abstract}
${ }^{1}$ Doctora en Psicología. Ayudante de Trabajos Prácticos Regular de la materia Teoría y Técnica de Exploración y Diagnóstico Psicológico. Investigadora Adjunta de la Universidad de la Defensa Nacional. Becaria de la Agencia Nacional de Promoción Científica y Tecnológica. E-mail: solgrin@yahoo.com.ar
\end{abstract}

${ }^{2}$ Licenciado en Psicología. Doctor en Humanidades Médicas. Jefe de Trabajos Prácticos en la cátedra Metodología de la Investigación, Facultad de Psicología, Universidad de Buenos Aires. Investigador Adjunto de la Universidad de la Defensa Nacional. Becario de la Agencia Nacional de Promoción Científica y Tecnológica. E-mail: azzarasergio@gmail.com

${ }^{3}$ Doctor y Profesor en Ciencias Antropológicas. Investigador en la Sección Etnología y Etnografía del Instituto de Ciencias Antropológicas de la Facultad de Filosofía y Letras, Universidad de Buenos Aires (UBA). Profesor en la Universidad Nacional de Lanús (UNLa). Profesor titular del seminario Antropología del Turismo en la Maestría de Economía y Gestión del Turismo, Facultad de Ciencias Económicas (FCE, UBA). Profesional de Apoyo en Formación. Becario de la Agencia Nacional de Promoción Científica y Tecnológica.

${ }^{4}$ Doctora en Psicología. Investigadora Independiente del Consejo Nacional de Investigaciones Científicas y Técnicas (CONICET). Profesora Adjunta de la Facultad de Psicología de la Universidad de Buenos Aires (UBA). Secretaria de Investigación de la Facultad del Ejército de la Universidad de la Defensa Nacional. Profesora de Posgrado en las Universidades de Buenos Aires (UBA), La Plata (UNLP) y Abierta Interamericana (UAI). Directora del proyecto D-TEC 0015/13.

Este trabajo contó con el subsidio de la Agencia Nacional de Promoción Científica y Tecnológica.

Facultad del Ejército, Universidad de la Defensa Nacional.

Buenos Aires, Argentina.

\section{Resumen}

Se realizó un estudio explicativo y transversal con el objetivo de analizar la efecti- vidad y los tiempos en la toma de decisiones al momento de brindar una primera ayuda psicológica (PAP) en las fases iniciales de un desastre en función del estilo y el proceso 
decisorio implicados. Se tomó una muestra no probabilística intencional de 165 voluntarios rescatistas de cuatro cuarteles de la zona de AMBA, se los dividió aleatoriamente en dos grupos (capacitados y no capacitados en PAP) y, a su vez, dichos grupos fueron divididos aleatoriamente respecto de la consigna impartida en la evaluación sobre el proceso decisorio a utilizar (decidir en función de lo que se piensa o de que lo que se siente). Asimismo, cada rescatista fue clasificado como "racional" o "afectivo" según el estilo decisorio urgente medido por el instrumento Bases for Urgent Decisions under Extreme Circumstances Inventory (BUDECI). Los resultados mostraron que los menos efectivos y más lentos fueron aquellos rescatistas capacitados con un estilo urgente decisorio afectivo, bajo una consigna que apelaba también a lo emocional. Este último grupo no presentó diferencias respecto de su efectividad cuando se lo comparó con el grupo de los no capacitados. La mayor efectividad la obtuvieron aquellos grupos que tenían un estilo o una consigna de tipo racional. La capacitación en PAP ha posibilitado la adquisición de estrategias de acción sencillas. Se concluye que el estilo decisorio y/o una inducción decisoria racionales favorecen una mayor efectividad de las acciones de PAP en las fases iniciales de un desastre.

Palabras clave: estilos decisorios; tiempos decisorios; efectividad; rescatistas; primera ayuda psicológica

\section{Abstract}

The protocol on Psychological First Aid (PFA) establishes a number of actions to take on victims who are in the area of an event. Through these actions, the victims are assisted and helped to feel calm but not passive. Regarding the implementation of PFA in disaster situations, the type of decision-making, analytic or intuitive, could represent an important factor in the effectiveness of the actions of aid provided by volunteers in emer- gencies and disaster situations. Modifications of a PFA protocol are presented to apply to victims in the early stages of a disaster; these modifications are intended to clear the life-saving area for the rescuer and reduce the risk of post-traumatic stress disorder (PTSD). Starting from the premises of the original model of Farchi, known as the Model of the $6 \mathrm{Cs}$, a simplified version for its application in Argentina was denominated the Model of the 4 Cs. In the same way, it is considered that, when assisting a person in a situation of crisis or disaster, the objective is to promote the activity of the prefrontal cortex and decrease the action of the limbic system. To do this, four guidelines are proposed: 1) Cognitive Communication, 2) Control and Challenge, 3) Commitment and 4) Continuity. Likewise, a PFA protocol based on the above mentioned model is presented. An explanatory and cross-sectional study was carried out with the objective of analyzing the effectiveness and the decision times when providing a PFA in the initial phases of a disaster, depending on the style and decision process involved. An intentional non-probabilistic sample, composed of 165 rescue volunteers from 4 fire stations in the AMBA area (Metropolitan Area and Greater Buenos Aires) and a headquarters of the Red Cross of the party of Tigre (San Fernando), was randomly divided into 2 groups (trained and untrained in PFA). Each volunteer was evaluated through the Software of the First Aid Interactive Psychological System (SIPAPSI); this consisted of a simulation of different crisis situations during which the participants must decide between different options presented in audiovisual format. The user must select the video that most closely approximates to what he or she would do in the presented situation. Then, the rescuers were randomly divided with respect to the slogan about the decision-making process used in the evaluation ("decide based on what you think", rational, or "decide based on what you feel", affective). The software SIPAPSI recorded the participant's response and the response time 
for each scene. Likewise, each volunteer was evaluated using the test Bases for Urgent Decisions under Extreme Circumstances Inventory (BUDECI). The BUDECI is composed of 8 elements that evaluate the decision-making style in unexpected and very important situations according to two dimensions: affective and rational. Rescuers were divided according to their urgent decision-making style: affective or rational. Therefore, the volunteers were classified according to whether or not they presented an adjustment between the decision-making style and the decision-making process induced by the slogan. This allowed us to study the influence of this adjustment on the effectiveness and decision time in disaster situations. The results showed that the least effective and, at the same time, slowest were those trained rescuers with an urgent affective decision style but induced under an emotional process. The PFA training has enabled the acquisition of simple action strategies in emergencies and disaster situations. It is concluded that the rational decision-making style or a rational decision induction favors a greater effectiveness of the PFA actions. These results allow us to build an optimal decisional profile for the rescuer's performance in crisis intervention tasks.

Keywords: decision-making styles; decision times; effectiveness; rescuers; first psychological aid.

\section{Introducción}

Una situación de desastre o de crisis implica un suceso negativo, a menudo imprevisto y brutal, que provoca destrucciones materiales y pérdidas humanas, y ocasiona un gran número de afectados y una desorganización social importante dado que la estructura social se rompe y algunas de sus funciones esenciales se ven inhabilitadas (Everly y Mitchell, 2008; North y Pfefferbaum, 2013; Silove, Steel y Psychol, 2006). En general, son situaciones extremas que ponen en riesgo y amenazan la integridad física y psicológica de las personas.
La importancia del estudio de las situaciones de desastre está justificada por los innumerables efectos que producen en la salud de una comunidad, ya que pueden provocar tanto trastornos físicos como psicológicos: ansiedad, depresión y el denominado trastorno de estrés postraumático (TEPT). Afrontar el suceso de la manera más adecuada permite al individuo sentir un menor número de conductas disruptivas, evitar las consecuencias en su salud mental, recuperarse y controlar la situación (Páez, Arroyo y Fernández, 1995), por lo que la intervención en el momento del hecho a través de formas de actuación eficaces para cada situación resulta central. Es fundamental que la persona afectada juegue un papel activo y sea parte de la solución del problema.

La asistencia psicológica temprana a afectados en una situación de desastre por parte de voluntarios rescatistas consiste en el cuidado psicológico para estabilizar y reducir los síntomas de estrés y la conducta disfuncional, de manera de lograr un estado de funcionamiento adaptativo y facilitar, además, el acceso a otro nivel de atención (Everly y Mitchell, 2008; Jarero, Artigas y Luber, 2011). Este tipo de intervención tiene cuatro objetivos centrales: (1) estabilizar el funcionamiento psicológico a través de la indagación de las necesidades básicas de los afectados y de la implementación de los recursos para poder satisfacerlas, promoviendo gradualmente su autonomía; (2) disminuir el estrés mediante la transmisión de seguridad, calma y contención; (3) recuperar el funcionamiento psicológico autónomo y adaptativo; y (4) facilitar el acceso a otros niveles de atención psicológica, en caso de que sea necesario (Everly, Barnett, Sperry y Links, 2010; Jarero, Artigas y Luber, 2011). Estos objetivos favorecen el restablecimiento de la calma y de la estabilización, la conexión con los demás y con los recursos para satisfacer las necesidades básicas materiales y psicológicas, incrementando la sensación de autoeficacia y de control (Benight y Bandura, 2004).

Siguiendo esta línea de abordaje, la Orga- 
nización Mundial de la Salud (OMS), en el año 2012, publicó una guía de Primera Ayuda Psicológica (PAP) para los rescatistas $\mathrm{y}$ trabajadores de campo que intervienen en situaciones de crisis o desastre. Se define la PAP como una respuesta de apoyo hacia una persona que está sufriendo y que necesita asistencia. Bajo esta premisa, la PAP se ocupa de brindar ayuda y apoyo de forma no invasiva, evaluar las necesidades y preocupaciones, ayudar a las personas a atender sus necesidades básicas, escucharlas sin presionar a los afectados para que hablen, reconfortándolos y ayudándolos a sentirse calmos, asistiéndolos para que accedan a información, servicios y apoyos sociales, y brindar protección respecto a ulteriores peligros. Dicha ayuda no tiene que ser brindada necesariamente por profesionales de la salud (OMS, 2012).

En nuestro medio, se ha modificado un protocolo de PAP (Azzollini et al., 2017, del original de M. Farchi, comunicación personal, 7 y 8 de agosto de 2012) que aplica a las personas afectadas en las fases iniciales de un desastre. Dicho protocolo tiene como principales objetivos despejar el área de trabajo del socorrista y disminuir el riesgo de TEPT. El modelo modificado consiste en cuatro premisas que orientan la acción del rescatista:

1) Comunicación cognitiva: se trata de activar la corteza prefrontal y disminuir la hiperactividad límbica a través de una interacción cognitiva sencilla entre el rescatista y el afectado.

2) Control y desafío: el objetivo que se persigue es que el implicado vaya adquiriendo la sensación de control a través de la toma de decisiones simples.

3) Compromiso: se propone disminuir la sensación de vulnerabilidad y soledad a través del planteo expreso de un pacto de acompañamiento.

4) Continuidad: apunta a disminuir la confusión de la persona ubicándola en el tiempo y el espacio, explicándole brevemente qué fue lo que ocurrió, qué está aconteciendo ahora y qué va a pasar después, a fin de contribuir a organizar la secuencia temporal de eventos.

De esta manera, se intenta lograr que el afectado salga de una situación de pasividad disfuncional y vulnerabilidad, y comience a adquirir sensaciones de autonomía y autoeficacia que le permitan lograr gradualmente un estado de actividad funcional, independencia y adaptación psicológica (Farchi et al., 2018).

Por todo lo expuesto, contar con un cuerpo de voluntarios rescatistas entrenados para realizar la PAP y colaborar con los equipos especializados en situaciones de desastre mejoraría la organización general de la tarea de rescate y reduciría las tasas de TEPT (de Leo et al., 2003).

Las habilidades que constituyen la PAP suponen un conocimiento técnico científicamente fundamentado que contempla destrezas y capacidades que se pueden enseñar y adquirir mediante un programa de capacitación. La PAP supone un perfil de competencias que son el conjunto de comportamientos observables que están causalmente relacionados con un desempeño óptimo en una tarea concreta (Pereda y Berrocal, 1999). Se han llevado adelante estudios controlados que han validado la eficacia de la capacitación en el rendimiento de rescatistas en PAP (Farchi et al., 2018).

Para llevar a cabo las cuatro acciones del modelo de PAP mencionadas (comunicación cognitiva, control y desafío, compromiso y continuidad), los rescatistas deben tomar decisiones a lo largo de todo el proceso, lo cual supone una tarea compleja teniendo en cuenta que se debe llevar a cabo en escenarios de elevada incertidumbre $\mathrm{y}$, por sobre todas las cosas, con un tiempo limitado para accionar.

En este sentido, el estudio de la relación entre los procesos de toma de decisión y los tiempos decisorios se ha basado en las teorías de los procesos duales (Kahnemany Frederick, 2002). Desde esta teoría, se han postulado dos procesos de razonamiento independientes y competitivos: un proceso automático, en gran parte inconsciente y poco preciso (Tipo 1), 
y un segundo proceso analítico-racional que conduce a resultados más certeros (Tipo 2) (Evans, 2012; Kahneman, 2003). El proceso Tipo 1 supone poco esfuerzo, rapidez y una fuerte dependencia del contexto, mientras que el de Tipo 2 funciona más lento y de manera secuencial (Evans y Stanovich, 2013).

Estudios recientes han mostrado que el proceso decisorio, ya sea Tipo 1 (intuitivo) o Tipo 2 (racional), puede afectar la efectividad en las tareas de rescate (Moreno Jiménez, Morett Natera, Rodríguez Muñoz y Morante Benadero, 2006). Por un lado, se han encontrado investigaciones que concluyen que las decisiones intuitivas suelen ser más eficaces que las racionales en contextos de alta incertidumbre (Azzollini, Depaula, Piñeyro y Torres, 2012), ya que las personas que utilizan el razonamiento consciente y deliberado no pueden recabar y procesar toda la información relevante $\mathrm{y}$, por lo tanto, toman decisiones menos favorables; o bien, que las decisiones intuitivas se benefician del uso de atajos cognitivos que permiten llegar a conclusiones más rápidamente $\mathrm{y}$ con una mayor sensación de certeza (Dijksterhuis, Bos, van der Leij y van Baaren, 2009; Dijksterhuis, Loran y Nordgren, 2006; Gigerenzer, 2007; Halberstadt, 2005; Halberstadt y Levine, 1999; Wilson y Schooler, 1991).

Sin embargo, Evans (2012) afirmó que las teorías previas basadas en los modelos duales suponen una serie de falacias: (a) que el procesamiento Tipo 1 por lo general conduce a respuestas erróneas (debido a sesgos cognitivos), mientras que el procesamiento Tipo 2 favorece respuestas correctas; (b) el procesamiento Tipo 1 se utiliza siempre y únicamente cuando los juicios se hacen rápidamente; (c) solo el procesamiento racional Tipo 2 es consciente. Para responder a estas falacias, Evans sostiene que la toma de decisión rápida puede corresponder tanto al procesamiento Tipo 1 como al Tipo 2. También, este autor afirma que cualquier proceso decisorio en el cual no haya influido el estado de ánimo del sujeto es racional, aunque haya sido no consciente, por lo cual los procesos Tipo 1 pueden ser también racionales. Además, diversos teóricos (Betsch, y Kunz, 2008; Grandori, 2015) están de acuerdo en que las decisiones correctas y rápidas pueden resultar de procesar grandes cantidades de información, incluso más grandes que la cantidad usada en el proceso secuencial, debido a la ventaja del procesamiento paralelo, aunque la heurística simple también puede utilizarse en esos juicios.

En este sentido, Cosentino, Azzollini, Depaula y Castillo (2017) sostuvieron que los juicios rápidos pueden basarse tanto en la emocionalidad como en la racionalidad, y que tomar decisiones rápidas no puede implicar, en sí mismo, tomar decisiones equivocadas. Según Cosentino et al. (2017), la toma de decisión urgente ante situaciones extremas está constituida por dos dimensiones. Por una parte, una dimensión que se denomina "Decisión Urgente Emocional-Afectiva" (DUa), referida a la decisión basada en una reacción subjetiva, relacionada principalmente con el estado emocional de la persona. Por otra parte, la dimensión que recibe el nombre de "Decisión Urgente Racional" (DUr), y se refiere a la decisión caracterizada por el procesamiento cognitivo de la información, el cual incluye la valoración de las ventajas y desventajas de la situación urgente ante la que se está, el cálculo de probabilidades de éxito o fracaso, y la planificación del comportamiento, entre otros tipos de cálculos mentales.

De acuerdo con esto, un estudio reciente ha mostrado una asociación positiva entre la efectividad de la acción del rescatista y la tendencia individual a tomar decisiones racionales en situaciones de urgencia (Azzollini, Depaula, Cosentino y Bail Pupko, 2018). Por otra parte, en una investigación previa llevada a cabo en una muestra constituida por peacekeepers, se constató que la tendencia individual a tomar decisiones racionales rápidas está asociada con un menor tiempo de reacción, y la de tomar decisiones afectivas, con una menor efectividad en situaciones de incertidumbre (Cosentino, Azzollini, Depaula y 
Castillo, 2016).

Sin embargo, por más que una persona tenga un estilo decisorio urgente predominante, no necesariamente va a utilizarlo en todas las situaciones que se le presenten, con lo cual sigue pendiente la pregunta sobre qué proceso decisorio es el más efectivo a la hora de las intervenciones de los rescatistas. El paradigma experimental de Bestch y Kunz (2008) propone la inducción de uno u otro proceso a partir de la consigna "decida en función de lo que piensa o en función de lo que siente", a fin de operacionalizar esos procesos.

A partir de esto, los autores introducen el concepto de ajuste decisional cuando la estrategia de decisión preferida (estilo) se ajusta a la estrategia efectivamente utilizada (proceso). Los sujetos suelen tomar decisiones sobre la base de sensaciones emocionales o bien utilizando la información concreta y el pensamiento racional, pero muchas veces será el contexto el que definirá o determinará el proceso decisorio utilizado. De esta manera, Betsch y Kunz (2008) plantean la existencia de una mayor efectividad en la respuesta cuando hay ajuste decisional entre el estilo y el proceso decisorio llevado adelante. Estos resultados, en consecuencia, destacan la importancia de considerar los efectos del ajuste entre el estilo y el proceso decisorio en los estudios en los que se induce la toma de decisiones. Asimismo, otros autores plantean la relevancia de indagar si el ajuste decisional también afecta la calidad de la toma de decisiones, por ejemplo, si podría conducir a juicios y decisiones objetivamente superiores (Dijkstra, van der Pligt y van Kleef, 2017).

Paralelamente al tipo de estilo decisorio, debe considerarse también el tiempo que el rescatista emplea para tomar dichas decisiones. En la literatura, la velocidad de procesamiento (y, por ende, el tiempo utilizado) que caracteriza los dos sistemas se ha postulado como una variable diferencial distintiva. Los procesos de Tipo 2, más lentos y deliberados, dependen de operaciones en serie que consumen tiempo y recursos y están limitados por la capacidad de la memoria de trabajo. En relación con esto, una investigación realizada en Argentina ha encontrado que los procesos decisorios intuitivos requerían de menor tiempo y estaban vinculados con recuerdos semánticos, en tanto que los procesos analítico-deliberados demandaban un mayor tiempo decisional, y estaban también vinculados con recuerdos autobiográficos (Azzollini et al., 2012).

El objetivo del presente trabajo es analizar la efectividad y los tiempos en la toma de decisiones al momento de brindar una PAP en las fases iniciales de un desastre en función del estilo y el proceso decisorio implicados. A tal efecto, se han planteado las siguientes hipótesis: (1) los rescatistas capacitados tomarán decisiones más efectivas y presentarán menores tiempos de decisión que los rescatistas no capacitados; (2) la efectividad y los tiempos decisorios están relacionados; (3) el tipo de estilo decisorio urgente y los tiempos decisorios explicarán la efectividad de la acción del rescatista; y (4) la existencia de ajuste decisional mejorará la efectividad de la decisión.

\section{Método}

\section{Diseño y muestra}

El estudio fue explicativo transversal de diferencias entre grupos.

La muestra fue no probabilística intencional. Estuvo conformada por 165 voluntarios rescatistas (66 mujeres y 99 varones) de cuatro cuarteles de la zona del Área Metropolitana y Gran Buenos Aires (AMBA) y una sede de la Cruz Roja del partido de Tigre (San Fernando), con una media de edad de 30.9 años $(D E=9.9)$. La muestra se dividió aleatoriamente en dos grupos. Por un lado, un grupo experimental integrado por 84 voluntarios (51 $\%)$ que recibieron una capacitación en PAP previamente a la evaluación (35 mujeres y 49 varones; $M=29.34$ años, $D E=9.22)$; por el otro lado, un grupo control integrado por 81 
voluntarios (49\%, 31 mujeres y 50 varones; $M=31.39$ años, $D E=10.74)$ que al momento de la evaluación aún no habían sido capacitados (se les dio una tarea distractora durante un tiempo equivalente a la duración de la capacitación).

\section{Procedimiento}

Se pactaron tres encuentros con los respon- sables de cada institución. En el primero se capacitó al grupo experimental y se le dio una tarea distractora al grupo control. En el segundo se realizó la evaluación de ambos grupos entre una a dos semanas posteriores -el experimental y el control- mediante el software SIPAPSI. En el último encuentro se concretó la capacitación al grupo control. Para una mejor comprensión del procedimiento explicado, se sugiere ver la Tabla 1.

Tabla 1

Procedimiento efectuado con el grupo control y el grupo experimental.

\begin{tabular}{ccc}
\hline & Grupo control & Grupo experimental \\
\hline Primer paso & Tarea distractora & Capacitación en PAP \\
Segundo paso & Evaluación SIPAPSI & Evaluación SIPAPSI \\
Tercer paso & Capacitación en PAP & ----------- \\
\hline
\end{tabular}

El SIPAPSI es un software que consta de 10 sets de cuatro videos cada uno, de los cuales hay una única opción correcta, que corresponde al protocolo de la PAP (véase apartado Instrumentos).

Al iniciar la evaluación del SIPAPSI, cada evaluado recibió una consigna inicial. A un grupo de participantes se les pidió que respondieran en base a lo que sentían y a otro grupo de participantes se les solicitó que respondieran en base a lo racional. Posteriormente, se administró el instrumento BUDECI (Bases for Urgent Decisions under Extreme Circumstances Inventory; ver apartado Instrumentos) que permitió conocer la tendencia decisoria urgente -afectiva o racional- de los participantes a la hora de responder a una situación de crisis, más allá de la consigna inicial recibida puntualmente al comienzo. La consigna inicial y la tendencia decisoria urgente (determinada por el BUDECI) permitieron clasificar la existencia o no del ajuste entre el estilo y el proceso decisorio para relacionarla con la efectividad (medida a través de la cantidad de respuestas correctas al SIPAPSI). Para ello, se dividió la muestra en cuatro grupos. El primer grupo, denomi- nado "Ajuste Emocional", estuvo integrado por aquellos evaluados con una tendencia decisoria urgente afectiva en el BUDECI y que, además, recibieron la consigna inicial de responder al SIPAPSI en base a lo que sentían, es decir, a lo emocional. El segundo grupo, denominado "Ajuste Racional", estuvo integrado por aquellos rescatistas que obtuvieron una tendencia decisoria urgente racional en el BUDECI y que, a su vez, siguieron la consigna inicial de responder al SIPAPSI en base a lo que pensaban, es decir, a lo racional. El tercer grupo, llamado "Desajuste Emocional", estuvo compuesto por rescatistas con tendencia decisoria urgente de tipo afectiva según el BUDECI a pesar de haber recibido una consigna inicial en la cual debían responder al SIPAPSI de acuerdo a lo que pensaban, es decir, a lo racional. Finalmente, el cuarto grupo, consignado como "Desajuste Racional", estuvo integrado por aquellos rescatistas que recibieron una consigna inicial de responder al SIPAPSI en base a lo que sentían, es decir lo emocional, y mostraron una tendencia decisoria urgente racional de acuerdo al BUDECI. Para una mejor comprensión, se sugiere ver la Tabla 2. 
Tabla 2

Denominación de los grupos de acuerdo al ajuste entre la consigna recibiday el estilo decisorio urgente.

\begin{tabular}{ccc}
\hline & $\begin{array}{c}\text { Recibió consigna } \\
\text { inicial }\end{array}$ & $\begin{array}{c}\text { Contestó al BUDECI } \\
\text { con un estilo decisorio }\end{array}$ \\
\hline Grupo "Ajuste Emocional" & Emocional & Emocional \\
Grupo "Desajuste Emocional" & Emocional & Racional \\
Grupo "Ajuste Racional" & Racional & Racional \\
Grupo "Desajuste Racional" & Racional & Emocional \\
\hline
\end{tabular}

Resulta importante aclarar que, con el SIPAPSI, al tratarse de un software, se pudo cronometrar el tiempo que demoró cada participante desde que se le presentaba cada situación a resolver hasta que, efectivamente, elegía la opción que consideraba la correcta entre las cuatro posibles. Esto permitió evaluar los tiempos de decisión promedio obtenidos por el grupo control y el grupo experimental.

Debido a la cantidad de variables presentes en este estudio, se ha elaborado la Tabla 3, la cual detalla cada una de ellas con su correspondiente operacionalización.

Tabla 3

Operacionalización de las variables presentes en el estudio.

\begin{tabular}{|c|c|}
\hline Variable & Operacionalización \\
\hline Efectividad & $\begin{array}{l}\text { Cantidad de respuestas correctas obtenidas en } \\
\text { el SIPAPSI. }\end{array}$ \\
\hline Tiempo de decisión & $\begin{array}{l}\text { Tiempo cronometrado desde que se le } \\
\text { presenta la situación a resolver al voluntario } \\
\text { hasta que elige la opción que considera } \\
\text { correcta entre los cuatro videos posibles. }\end{array}$ \\
\hline Estilo decisional & Puntaje obtenido en el BUDECI. \\
\hline Ajuste decisional & $\begin{array}{l}\text { Coincidencia o no entre la consigna inicial } \\
\text {-racional o emocional- y el estilo decisional } \\
\text { urgente obtenido por el BUDECI. }\end{array}$ \\
\hline
\end{tabular}

Por otra parte, resulta pertinente aclarar que, en todos los casos, los participantes firmaron un consentimiento informado y no recibieron retribución alguna. La capacitación tuvo una duración de dos horas aproximadamente, y se dividió en tres módulos (dos teóricos y uno práctico). En un primer módulo se desarrollaron conceptos básicos: respuesta de estrés y estructuras cerebrales involucradas. En el segundo módulo se abordaron cuestiones referidas a la PAP y al protocolo basado en el modelo de las 4 Cs (Azzollini et al., 2017; Farchi, 2012). En el último módulo -de índole práctico- se promovió el espacio para que los rescatistas pusieran en práctica lo 30 Decisiones y efectividad en la primera a adquirido en los dos módulos teóricos precedentes a través de dramatizaciones de situaciones que se repiten en su labor cotidiana. Se entregaron certificados de asistencia a cada rescatista y un certificado a la institución. Asimismo, se acompañó la capacitación con material gráfico que resumía los principales aspectos de la PAP.

\section{Instrumentos}

Se utilizó el software Sistema Interactivo de Primera Ayuda Psicológica (SIPAPSI) (Depaula, Torres, Piñeyro, Cosentino, Clotet y Castillo, 2012), diseñado para evaluar las
psicológica. INTERDISCIPLINARIA, 2020, 37(2), 23-37 
respuestas de PAP en los usuarios. Consiste en la simulación de distintas situaciones de crisis durante las cuales los participantes deben decidir entre diferentes opciones presentadas en formato audiovisual. Al comenzar, se le otorga al evaluado una consigna: que responda en base a lo que siente, es decir, a lo emocional o que responda en base a lo que piensa, es decir, lo racional. Posteriormente, se le presentan al usuario 10 sets de cuatro videos con simulaciones de las respuestas de los voluntarios hacia las personas recientemente afectadas por una catástrofe; en cada set hay una única respuesta correcta en función de los 10 pasos del protocolo. El usuario debe seleccionar el video que más se aproxime a lo que haría en la situación representada. Para cada set, se realiza la presentación de los cuatro videos en sucesión, sin interrupción o intervención posible por parte del usuario. Luego, el participante tiene la posibilidad de revisar todos o algún video nuevamente. El SIPAPSI registra las respuestas correctas y el tiempo de decisión promedio total de cada voluntario (es decir, el tiempo que tarda el rescatista desde que se le presentan las cuatro opciones de respuesta hasta que efectivamente elige una, dividido por las 10 opciones de presentación). El SIPAPSI tiene evidencia de validez de contenido, y cuenta entre los jueces expertos con el Dr. Moshe Farchi, quien ha desarrollado el protocolo de intervención PAP basado en su modelo original de las 6 Cs (Farchi et al., 2018).

Luego de los 10 conjuntos de videos, se inicia la presentación del BUDECI. El BUDECI está integrado por ocho ítems que valoran el estilo decisorio en situaciones nuevas, inesperadas y muy importantes de acuerdo a dos dimensiones -decisión urgente afectiva o racional- (Cosentino et al., 2017). La primera dimensión, la DUa (véase apartado Introducción), refiere a la reacción subjetiva relacionada principalmente con el estado emocional de la persona. Por su parte, la segunda dimensión, DUr (véase apartado Introducción), se caracteriza por el procesa- miento cognitivo de la información, e incluye la valoración de las ventajas y desventajas de la situación, el cálculo de probabilidades y la planificación del comportamiento, entre otras cuestiones. La escala cuenta con un formato de respuesta Likert que va del 1 (totalmente falso) al 7 (totalmente verdadero). Este instrumento cuenta con evidencia de validez referida a los aspectos estructurales, convergentes y de grupos contrastados. A su vez, los valores de confiabilidad de las dimensiones fueron: para DUa, $\alpha=.87$, y para DUr, $\alpha=.88$.

\section{Resultados}

En principio, y para poner a prueba la primera hipótesis, se analizó la existencia de diferencias estadísticamente significativas entre el grupo que recibió la capacitación en PAP y el grupo que no la recibió. Lo hipotetizado en el presente estudio refería a que los rescatistas capacitados (grupo experimental) deberían tomar decisiones más efectivas que los no capacitados (grupo control). Se llevó a cabo una prueba $t$ para muestras independientes. Los resultados mostraron una diferencia estadísticamente significativa de la efectividad (medida con el número de respuestas correctas al SIPAPSI) entre ambos grupos. Se pudo observar que el número total de respuestas correctas en el grupo experimental fue de 72 , mientras que en el grupo control fue de 43. por lo tanto, el grupo experimental presentó un promedio significativamente mayor de respuestas correctas que el grupo control (capacitados: $M=6.88, D E=$ 1.81; no capacitados: $M=5.14, D E=1.57 ; t=$ 6.59; gl. $=163 ; p=.01)$.

Luego, y siguiendo con la primera hipótesis, se buscó conocer si existían diferencias en los tiempos de decisión promedio medidos a través del software SIPAPSI de acuerdo a si los voluntarios recibieron capacitación en PAP (grupo experimental) o no (grupo control). Con respecto a esto, se había hipotetizado que el grupo experimental, al haber sido capacitado en un protocolo de PAP, que 
detallaba los pasos a seguir en una situación de crisis (los cuales posteriormente se evaluaban en el software SIPAPSI), deberían presentar menores tiempos de decisión que los rescatistas del grupo control. Se realizó otra prueba $t$ para muestras independientes. Los resultados no estuvieron en consonancia con la hipótesis, es decir, no existieron diferencias significativas entre las medias de los tiempos de decisión promedio demandados por ambos grupos. Se indagó entonces si la efectividad (medida a través de las respuestas correctas brindadas en el SIPAPSI) se encontraba asociada a los tiempos de decisión, segmentando la muestra entre capacitados y no capacitados. Se pudo observar una correlación inversa y estadísticamente significativa entre la efectividad y los tiempos de decisión únicamente en el grupo de los capacitados $(r$ $=-.26 ; p=.02$ ). Esto significa que aquellos voluntarios capacitados que mostraron una mayor efectividad fueron a su vez más rápidos en tomar la decisión.

De acuerdo a la segunda hipótesis, se analizó la correlación entre la efectividad y el estilo decisorio urgente afectivo, por un lado, y racional, por el otro, mediante una $r$ de Pearson. Los resultados mostraron que existe una correlación inversa y estadísticamente significativa entre la efectividad y el estilo decisorio urgente afectivo $(r=-.27 ; p=.04)$ solamente en el grupo de voluntarios capacitados $(n=84)$. Estos resultados sugieren que los capacitados con un menor estilo decisorio urgente afectivo muestran mayor cantidad de respuestas correctas al software SIPAPSI.

Posteriormente, y en relación con la tercera hipótesis, se quiso conocer si el estilo decisorio empleado y el tiempo de decisión demandado por el voluntario podían explicar la efectividad en las respuestas brindadas. Con tal motivo, se llevó a cabo una regresión lineal en ambas muestras (capacitados y no capacitados) habiéndose verificado previamente que los residuos poblacionales eran independientes y se distribuían normalmente con una media 0 y con una varianza común para todos. De esta forma, para el desarrollo del modelo se utilizaron, entonces, como variables explicativas el tiempo de decisión y el estilo decisorio, y, como variable criterio, la efectividad. La capacidad de predicción del modelo resultante fue significativa solo para el grupo de capacitados, explicando el $11.5 \%$ de la varianza. La prueba de ANOVA fue estadísticamente significativa $[F(3,80)=3.27 ; p$ $=.02]$.

Tabla 4

Coeficientes y niveles de significación para las variables incluidas en el modelo.

\begin{tabular}{ccccc}
\hline Modelo & $\mathrm{B}$ & $\begin{array}{c}\text { Error } \\
\text { estándar }\end{array}$ & $\mathrm{T}$ & Sig. \\
\hline Constante & 7.46 & 1.31 & 5.67 & $.000^{* *}$ \\
Tiempo de decisión & -3.37 & .00 & -1.79 & .07 \\
Estilo afectivo & -.06 & .03 & -2.01 & $.04^{*}$ \\
Estilo racional & .02 & .06 & .47 & .63 \\
\hline
\end{tabular}

$* \mathrm{p}<.05$

$* * \mathrm{p}<.001$

Finalmente, se puso a prueba la última hipótesis del presente estudio que planteaba que la existencia de ajuste decisional mejoraría la efectividad de la decisión conforme con lo planteado por Betsch y Kunz (2008). Se analizó el efecto del ajuste entre la consigna y el estilo decisorio sobre la efectividad a través de un ANOVA, segmentando la muestra en rescatistas capacitados y no capacitados, utilizando como variable dependiente la efecti- 
vidad medida por las respuestas correctas al SIPAPSI y como criterio una variable categorizada en cuatro grupos: (1) ajuste emocional; (2) ajuste racional; (3) desajuste emocional y (4) desajuste racional (véase Tabla 2).

Se observaron diferencias estadísticamente significativas en las medias de los cuatro grupos, pero únicamente en los capacitados $[F$ $(\mathrm{gl})=4.05(3) ; p=.01]$. Las comparaciones post-hoc indicaron que el grupo que mostró diferencias significativas fue el de ajuste emocional respecto de los otros tres grupos: ajuste racional $(p=.03)$, desajuste emocional $(p=.005)$ y desajuste racional $(p=.006)$. En la Figura 1 se puede ver que el grupo ajuste emocional fue el que menos efectividad mostró a la hora de responder al SIPAPSI aun habiendo sido capacitado previamente.

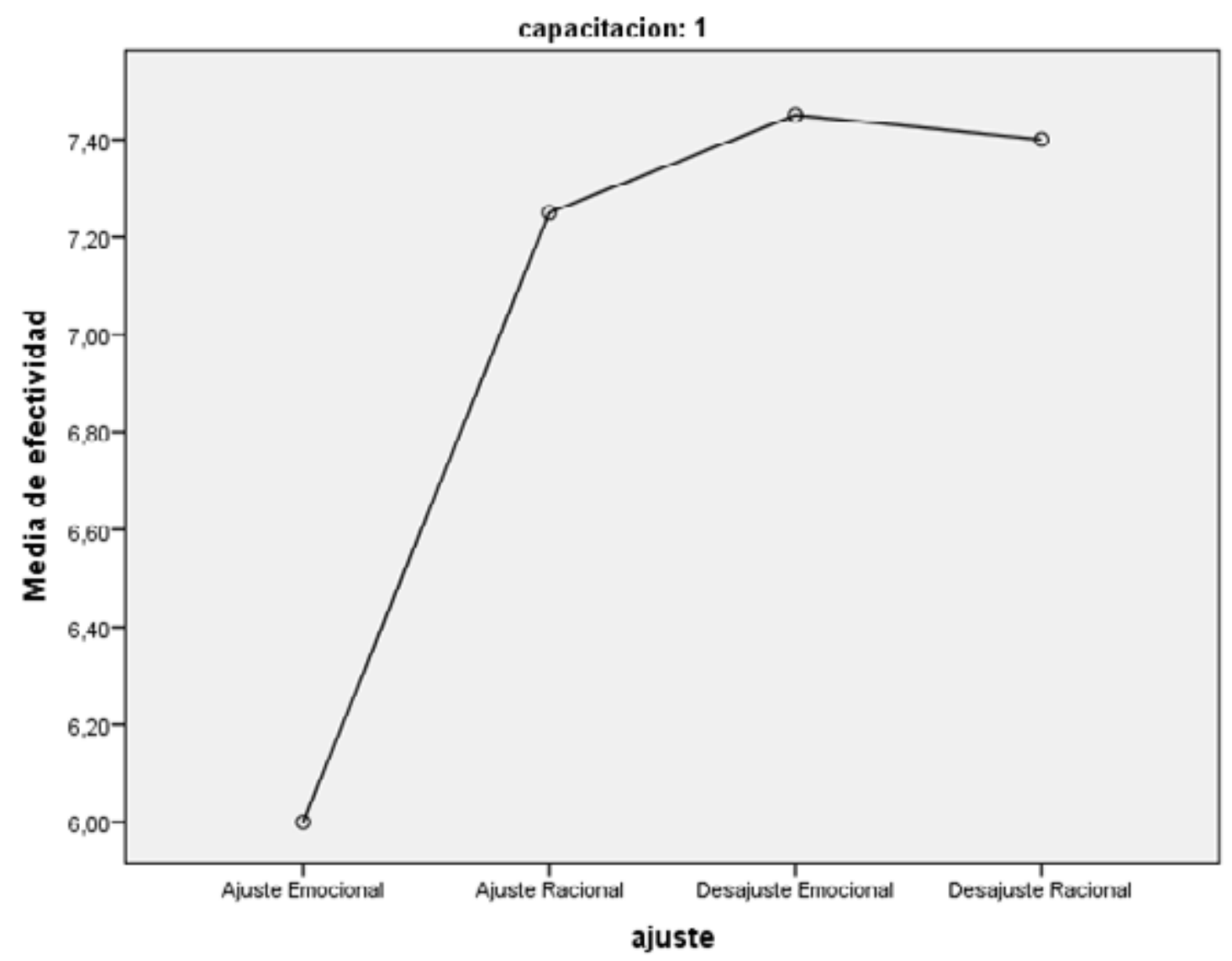

Figura 1. Efectividad de acuerdo al ajuste entre consigna y estilo decisorio urgente en capacitados.

\section{Discusión}

En relación con la primera hipótesis, al comparar ambos grupos -capacitados y no capacitados- se puso en evidencia la eficacia de la capacitación en PAP. Es decir, los rescatistas voluntarios que recibieron el entrenamiento en PAP mostraron un número promedio significativamente mayor de respuestas correctas en la toma de decisiones sobre el software SIPAPSI que aquellos que no habían recibido dicho entrenamiento. De esta forma, se puede afirmar que dicha capacitación en PAP ha posibilitado la adquisición de estrategias de acción sencillas en situaciones de urgencia (Farchi, 2012, 2018).

Sin embargo, y en disonancia con dicha hipótesis, es de interés notar que no se encontraron diferencias significativas en el tiempo promedio de decisión entre rescatistas capacitados y no capacitados. Esto pudo deberse a que, para que los voluntarios entrenados 
puedan actuar más rápido, deben primero internalizar el protocolo impartido en la capacitación (Klein, 1998, 2008; Klein y Crandall, 1996), lo cual solo se logra mediante su práctica (Altman, 2012; Betsch y Glöckner, 2010; Depaula y Azzollini, 2016; Easen y Wicockson, 1996; Gladwell, 2005; Lunenburg, 2010; Simon, 2000). En este sentido, debe tenerse en cuenta que la evaluación mediante el software SIPAPSI se llevó a cabo entre una y dos semanas luego de la capacitación, tiempo insuficiente para llevar a cabo dicho proceso de incorporación y automatización del modelo (Betsch, 2008). Esto podría explicar por qué el grupo de capacitados, si bien más efectivo, no mostró una mayor rapidez en sus decisiones al ser evaluado. Sin embargo, al interior de dicho grupo se observó que, cuanta mayor efectividad mostraban, más veloces eran a la hora de escoger la opción correcta. En este sentido, la decisión rápida sustentada en la activación instantánea de un patrón de reconocimiento ligado a experiencias previas (modelo PAP) que se conectan (matching process; Dane y Pratt, 2007) a la situación actual (situaciones planteadas mediante la evaluación con el SIPAPSI) (Klein, 1998, 2008; Klein y Crandall, 1996) podría explicar esta tendencia en el grupo de capacitados solamente.

Siguiendo con la segunda hipótesis, se encontró que las decisiones urgentes de base afectiva se asocian de forma inversa al número de respuestas correctas obtenidas en el SIPAPSI en general y en el grupo experimental en particular. Dichos resultados se encuentran en consonancia con un estudio previo también llevado a cabo en Argentina (Azzollini, Depaula, Cosentino y Bail Pupko, 2018). En dicha investigación, se pudo constatar que las respuestas dadas a partir de la inducción racional resultaron más efectivas que las brindadas bajo una consigna emocional. Si bien en estudios realizados en otros países se encontró que las decisiones de tipo intuitivo presentan mayor eficacia que las racionales en contextos de elevada incertidumbre (Gigerenzer, 2007; Halberstadt,
2005; Halberstadt y Levine, 1999), es necesario recordar que la intuición entendida como proceso racional no consciente (Evans, 2012) podría estar ratificando los actuales resultados obtenidos en el presente estudio.

Asimismo, y según la tercera hipótesis, se determinó un modelo predictivo en el cual solo el estilo decisorio afectivo predijo una menor efectividad. En ese sentido, se deberá hacer uso de procesos analíticos y deliberativos para evaluar si es factible que una experiencia pasada pueda ser aplicada en el contexto actual (Harteis y Billett, 2013). Es decir, el sistema de procesamiento racional permite el acceso a grandes cantidades de experiencia, sin embargo esto solo es útil si el decisor evalúa que la situación que enfrenta puede ser asimilada a los patrones y modelos almacenados en la memoria de largo plazo (Gladwell, 2005; Lunenburg, 2010). Otros autores (e.g. Gladwell, 2005; Lunenburg, 2010) señalan que aquellos que tienen la capacidad de tomar decisiones eficaces inmediatas y precisas basadas en "sensaciones" son expertos en el tema en cuestión y han pasado años desarrollando conocimientos y habilidades a través de la práctica, la repetición y la experiencia.

Por último, en relación con la última hipótesis, que se proponía analizar el efecto del ajuste entre la consigna y el estilo decisorio urgente sobre la efectividad, los resultados mostraron que solo el grupo de rescatistas capacitados ajustados emocionalmente fue significativamente menos efectivo que los otros tres grupos (ajuste racional; desajuste emocional y desajuste racional). Este resultado contradice el modelo de Betsch y Kunz (2008), según el cual la existencia o no de ajuste decisional debería influir en la efectividad. Esto puede explicarse debido a que si a aquellos voluntarios con un estilo decisorio urgente emocional se les solicita que respondan en base a lo que sienten, esto potencia las dificultades que conlleva dicho estilo en las acciones de rescate (Azzollini, Depaula, Cosentino y Bail Pupko, 2018). Análogamente, así como en los afectados por una catástrofe el compo- 
nente emocional favorece la hiperactivación límbica y promueve la aparición de TEPT, en los rescatistas disminuye la efectividad en las acciones del protocolo de PAP (Farchi, 2018). Lo que resulta interesante es que el grupo ajuste emocional resultó ser el menos eficaz de los cuatro grupos. Esto quiere decir que, así sea el estilo o la consigna, el elemento racional favorece una mayor efectividad (Depaula y Azzollini, 2016; Klein, 2005).

El juicio es un importante elemento en la intervención en situaciones de desastre. Los rescatistas tienen que tomar decisiones urgentes cuando entran en contacto con un individuo en situación vulnerable afectado por una catástrofe. Por lo tanto, la toma de decisión, en tanto componente predisponente del comportamiento a realizar ante contextos de incertidumbre y riesgo, es crucial. Este estudio aporta información para la determinación de un perfil que potencie la efectividad de las acciones ante una crisis. Conocer este perfil individual les permitirá a las instituciones determinar previamente qué elementos de recursos humanos se podrían disponer rápidamente en el terreno para asistir psicológicamente a personas en una catástrofe. Si bien este trabajo ha abordado el efecto de los estilos decisionales urgentes y de su ajuste con los procesos decisorios efectivamente inducidos sobre la efectividad de la acción, es indispensable analizar en trabajos ulteriores otras variables psicológicas que permitan clarificar aún más el perfil decisorio más adecuado del rescatista.

En conclusión, estos resultados permiten ir armando un perfil decisional óptimo del rescatista en el desempeño de tareas de intervención en crisis, así como inducirlo a realizar la PAP bajo una consigna racional que favorezca la efectividad. Empero, se debería tener en cuenta para futuras líneas de investigación la realización de estudios longitudinales en los cuales se pueda llevar a cabo un seguimiento de la aplicación concreta por parte de los voluntarios del protocolo de PAP, impartido en las capacitaciones, en el campo de acción.
Esto podría echar luz sobre los procesos de cristalización de los conocimientos a través de la experiencia y la puesta en acto de lo adquirido teóricamente.

Finalmente, el estudio presenta dos limitaciones principales. Por un lado, la muestra es pequeña por lo cual debería aumentarse su tamaño para así obtener resultados más confiables y de mayor fuerza estadística. Por otro lado, su baja validez ecológica por lo cual resultaría esclarecedor contrastar los presentes resultados, obtenidos en un ambiente artificial, con escenarios de acción reales, lo cual -sin duda- incrementaría la validez ecológica de la presente investigación. Pese a esto y entendiendo lo difícil que puede resultar llevar a cabo un estudio en el momento mismo en el cual ocurre la situación de crisis, el presente trabajo otorga conclusiones válidas sobre el mejor accionar.

\section{Referencias bibliográficas}

Altman, M. (2012). Implications of behavioral economics for financial literacy and public policy, The Journal of Socio-Economics, 41(5), 677-690.

Azzollini, S., Azzara, S., Depaula, P., Cosentino, A. y Grinhauz, A. (2017). Modification of the First Psychological Assistance Protocol in Situations of Crisis. Acta Psiquiátrica y Psicológica De América Latina, 63(2), 139-147.

Azzollini, S. C., Depaula, P. D., Piñeyro, D. R. y Torres, J. A. (2012). Incidencia del estilo decisorio predominante y el grado de incertidumbre cultural en la efectividad de las decisiones tomadas por militares voluntarios en misiones de paz. Revista Argentina de Ciencias del Comportamiento, 4(3), 13-21.

Azzollini, S., Depaula, P. D., Cosentino, A. C. y Bail Pupko, V. (2018). Applications of Psychological First Aid in disaster and emergency situations: its relationship with decision making. Athens Journal of Social Sciences, 5(2), 201-213.

Benight, C. C. y Bandura, A. (2004). Social cognitive theory of posttraumatic recovery: The role 
of perceived self-efficacy. Behaviour Research and Therapy, 42(10), 1129-1148.

Betsch, T. (2008). The nature of intuition and its neglect in research on judgment and decision making. En H. Plessner, C. Betsch y T. Betsch (Eds.), Intuition in judgment and decision making (pp. 3-22). Mahwah, NJ: Lawrence Erlbaum.

Betsch, T. y Glöckner, A. (2010). Intuition in Judgment and Decision Making: Extensive Thinking Without Effort. Psychological Inquiry, 21(4), 279-294. https://doi.org/10.1080/1047 840X.2010.517737

Betsch, C. y Kunz, J. J. (2008). Individual Strategy Preferences and Decisional Fit. Journal of Behavioral Decision Making, 21, 532-555. https://doi.org/10.1002/bdm.600

Cosentino, A. C., Azzollini, S. C., Depaula, P. D. y Castillo, S. E. (2016). Toma de decisión según racionalidad/afectividad, entrenamiento y saturación cultural en situaciones multiculturales: Un estudio experimental con soldados para la paz Interdisciplinaria, Revista de Psicología y Ciencias Afines, 33(2), 283-298. https://doi. org/10.16888/interd.2016.33.2.6

Cosentino, A. C., Azzollini, S. C., Depaula, P. D. y Castillo, S. E. (2017). Assessment of the affective and rational bases for urgent decision-making under extreme circumstances. Análise Psicológica, 35(4), 543-556.

Dane, E. y Pratt, M. G. (2007). Exploring intuition and its role in managerial decision making. Academy of Management Review, 32(1), 33-54. https://doi.org/10.5465/amr.2007.23463682

De Leo, G., Ponder, M., Molet, T., Fato, M., Thalmann, D., Magnenat-Thalmann, N., Bermano, F. y Beltrame, F. (2003). A Virtual Reality System for the Training of Volunteers Involved in Health Emergency Situations. Cyber Psychologyy Behavior, 6(3), 267-274.

Depaula, P. D. y Azzollini, S. C. (2016). Efectividad decisoria en ambientes culturales diversos y experiencias previas en misiones de paz: Un estudio correlacional con cascos azules argentinos. Psicodebate, 6(1), 83-108. https://doi.org/10.18682/pd.v16i1.567

Depaula, P., Torres, J. A., Piñeyro, D. R., Cosen- tino, A. C., Clotet, C. I. y Castillo, S. (2012). Desarrollo de un software para la evaluación de la toma de decisiones en contextos de incertidumbre cultural. IV Congreso Internacional de Investigación y Práctica Profesional en Psicología, XIX Jornadas de Investigación, VIII Encuentro de Investigadores en Psicología del MERCOSUR. Facultad de Psicología, Universidad de Buenos Aires. Buenos Aires, Argentina.

Dijksterhuis, A., Bos, M. W., van der Leij, A. y van Baaren, R. B. (2009). Predicting Soccer Matches After Unconscious and Conscious Thought as a Function of Expertise. Psychological Science, 20(11), 1381-1387. https://doi. org/10.1111/j.1467-9280.2009.02451.x

Dijksterhuis A., Loran F. y Nordgren, L.F. (2006). A theory of unconscious thought. Perspectives on Psychological Science, 1(2), 95-109.

Dijkstra, K. A., van der Pligt, J. y van Kleef, G. A. (2017). Fit between decision mode and processing style predicts subjective value of chosen alternatives. European Journal of Social Psychology, 47, 72-81. https://doi. org/10.1002/ejsp. 2217

Evans, J. S. B. T. (2012). Dual-Process theories of deductive reasoning: Facts and fallacies. En K.J. Holyoak y R.G. Morrison (Eds.), The Oxford handbook of thinking and reasoning (pp. 115-133). New York, NY, US: Oxford University Press. http://dx.doi.org/10.1093/ oxfordhb/9780199734689.013.0008

Evans, J. S. B. T. y Stanovich, K.E. (2013). Dual-process theories of higher cognition: Advancing the debate. Perspectives on Psychological Science, 8(3), 223-241.

Everly Jr, G. S., Barnett, D. J., Sperry, N. L. y Links, J. M. (2010). The use of psychological first aid (PFA) training among nurses to enhance population resiliency. International Journal of Emergency Mental Health, 12(1), 21-31.

Everly, G. S. y Mitchell, J. T. (2008). Integrative crisis intervention and disaster mental health. Ellicott City, MD: Chevron Publishing Corp

Farchi, M., Levy, T. B., Gershon, B. B., Hirsch-Gornemann, M. B., Whiteson, A. e 
Idron, Y. (2018). The SIX Cs model for Immediate Cognitive Psychological First Aid: From Helplessness to Active Efficient Coping. International Journal of Emergency Mental Health and Human Resilience, 20(2), 1-12.

Gigerenzer, G. (2007). Gut feelings: The intelligence of the unconscious. London: Penguin Books.

Gladwell, M. (2005). Blink: The power of thinking without thinking. New York, NY: Little, Brown.

Grandori, A. (2015). Heuristics as methods: Validity, reliability and velocity. En E. Ippoliti (Ed.), Heuristic reasoning (pp. 147-161). New York, NY, US: Springer International Publishing Switzerland. https://doi.org/10.1007/978-3319-09159-4 7

Halberstadt, J. (2005). Featural shift in explanationbiased memory for emotional faces. Journal of Personality and Social Psychology, 88, 38-49.

Halberstadt, J. B. y Levine, G. M. (1999). Effects of Reasons Analysis on the Accuracy of Predicting Basketball Games 1. Journal of Applied Social Psychology, 29, 517-530.

Harteis, C. y Billett, S. (2013). Intuitive expertise: Theories and empirical evidence. Educational Research Review, 9, 145-157.

Jarero, I., Artigas, L. y Luber, M. (2011). The EMDR protocol for recent critical incidents: Application in a disaster mental health continuum of care context. Journal of EMDR Practice and Research, 5(3), 82-94.

Kahneman, D. (2003). A perspective on judgment and choice: mapping bounded rationality. American Psychologist, 58(9), 697.

Kahneman, D. y Frederick, S. (2002). Representativeness revisited: Attribute substitution in intuitive judgment. Heuristics and biases: The psychology of intuitive judgment, 49, 81 .

Klein, G. (1998). Sources of Power: How People Make Decisions. Cambridge, MA: MIT Press.

Klein, G. (2005). The power of intuition: How to use your gut feelings to make better decisions at work. New York, NY: Knopf Doubleday.

Klein, G. (2008). Naturalistic Decision Making. Human Factors, 50(3), 456-460. https://doi. org/10.1518/001872008X288385

Klein, G. y Crandall, B. (1996) Recognition-Primed Decision Strategies. Alexandria, VA: US Army Research Institute for the Behavioral and Social Sciences.

Lunenburg, F. C. (2010). The power of intuition: How to use your gut feelings to make better managerial decisions. International Journal of Management, Bisiness, and Administration, 13(1), 1-5.

Moreno Jiménez, B., Morett Natera, N. I., Rodríguez Muñoz, A. y Morante Benadero, M. E. (2006). La personalidad resistente como variable moduladora del síndrome de burnout en una muestra de bomberos. Psicothema, 18(3), 413.

North, C. S. y Pfefferbaum, B. (2013). Mental health response to community disasters: a systematic review. Jama, 310(5), 507-518.

Organización Mundial de la Salud (OMS) (2012). Guía para trabajadores de campo. Ginebra: Nonserial Publications.

Páez, D., Arroyo, E. y Fernández, I. (1995). Catástrofes, situaciones de riesgo y factores psicosociales. Mapfre Seguridad, 57, 43-55.

Pereda, S. y Berrocal F. (1999). Gestión de Recursos Humanos en Competencias. Madrid: Centro de Estudios Ramón Areces.

Silove, D., Steel, Z. y Psychol, M. (2006). Comprender las necesidades psicosociales de la comunidad después de un desastre: Implicaciones para los servicios de salud mental. Revista de Medicina de Posgrado, 52(2), 121.

Simon, H. (2000). Barriers and bounds to Rationality. Structural Change and Economic Dynamics, 11, 243-253.

Wilson, T. D. y Schooler, J. W. (1991). Thinking too much: Introspection can reduce the quality of preferences and decisions. Journal of Personality and Social Psychology, 60, 181-192.

Recibido: 6 de julio de 2018 Aceptado: 29 de julio de 2020 